\title{
Research Article \\ Conductive Inks with 3D Conductive Networks of Polyaniline Crystals Nanofibers
}

\author{
Dawei $\mathrm{Li}^{1}$, Yulun Tao ${ }^{1,3,{ }^{*}}$, Shun $\mathrm{Yao}^{1}$, Wenxiang Tian ${ }^{1}$, Guihui Wang ${ }^{1}$, Baoyu $\mathrm{Li}^{1}$, Wuji Wang ${ }^{1}$, Shuo $\mathrm{Li}^{1}$, \\ Jinian Yang ${ }^{1}$, Qingbo $\mathrm{Yu}^{1}$, Shuxing $\mathrm{Wu}^{2}$, Il Kim ${ }^{3}$ \\ ${ }^{1}$ Department of Materials Science and Engineering, Anhui University of Science and Technology, Huainan 232001, PR China \\ ${ }^{2}$ School of Chemical Engineering and Light Industry, Guangdong University of Technology, Guangzhou 510006, PR China \\ ${ }^{3}$ Department of Polymer Science and Engineering, Pusan National University, Busan, South Korea
}

\section{ARTICLE INFO}

Article History

Received 14 December 2020

Accepted 17 March 2021

\section{Keywords}

Crystal polyaniline

nanofibers

citric acid

conductive ink

\begin{abstract}
A series of Polyaniline (PANI) nanoparticles doped with citric acid were synthesized by situ polymerization. It is interesting that uniform nanofibers with the diameter of $50 \mathrm{~nm}$ formed under a proper mass ratio (aniline:citric acid) of 5:4, which forms 3D conductive networks. XRD patterns show the nanofibers have sharp peaks, corresponding to high crystallinity. The nanofibers were selected for preparation of PANI ink with Polyethylene Glycol (PEG, Mn =698), Sodium Polystyrene Sulfonate (PSS, Mn = 70,000). The good electric conductivity of PANI conductive ink is $6.49 \times 10^{-3} \mathrm{~S} / \mathrm{cm}$, where PANI inks of Anhui University of Science and Technology university was stamped in A4 paper for good printing performance acting as electronic circuit line turning on a LED light. Moreover, the effects such as the content of citric acid and PSS on the conductivity of PANI powder and also discussed.
\end{abstract}

(C) 2021 The Authors. Published by Atlantis Press B.V. This is an open access article distributed under the CC BY-NC 4.0 license (http://creativecommons.org/licenses/by-nc/4.0/).

\section{INTRODUCTION}

Conductive polymer inks [1-5] are attractive for practical applications such as electronic circuit line, organic electrodes, sensors and capacitors, due to their printing performance, conductivity and chemical properties. Generally, nanofibers [6-9] are better conductive materials for inks because they can easily disperse and form $3 \mathrm{D}$ conductive networks $[10,11]$. However, the conductivity and stability of polymer inks are still not very high owing to disordered molecular chains of nanofibers [12-16]. Therefore, investigations in improving crystallinity of the nanofibers is a hot spot in preparation of high quality conductive polymer inks. Conductive ink generally refers to metal nanoparticles, metal nanowires, or transparent conductive oxide materials, such as indium tin oxide. These materials have high electrical conductivity, but they also have significant limitations. The main problem with total cost of ownership is the scarcity of available materials available to meet the continuous demand of global consumers, coupled with high production costs and poor mechanical flexibility may be a problem, however, recent progress reports indicate that total cost of ownership use is well tolerable to bending. Metal nanoparticles and nanowires are typically made up of metals such as gold, silver and copper, which are highly conductive but costly.

Here, we report a general route to create a series of Polyaniline (PANI) crystal nanofibers. The method takes place of room temperature and does not depend on any specific agents, equipment or heating, cooling and complex procedures, which is especially

"Corresponding author. Email: williamtao795322@163.com suitable for producing bulk quantities of high-quality and lowcost PANI crystal nanofibers for conductive polymer inks. The idea of preparing crystal nanofibers of PANI is very interesting and could potentially lead to significant advancements in polymer crystallization.

\section{EXPERIMENTAL SECTION}

All of the reagents including aniline, citric acid and Ammonium Persulfate (APS) are of analytical grade. They were used in experiments without further purification. Double distilled water was used throughout the experiment to prepare the solutions. Aniline, citric acid and APS were all purchased online. Aniline also known as anilin, anilin oil, amino benzene, molecular formula: $\mathrm{C}_{6} \mathrm{H}_{7} \mathrm{~N}$. Colorless oily liquid. Slightly soluble in water, soluble in ethanol, ether and other organic solvents. Aniline is one of the most important amines. Mainly used in the manufacture of dyes, drugs, resins, but also can be used as rubber vulcanization accelerator. It can also be used as a black dye itself. Citric acid is an important organic acid, the appearance is colorless crystal, odorless, has a strong sour taste, easily soluble in water. According to its water content, it is divided into monohydrate citric acid and anhydrous citric acid. APS, chemical formula: $\left(\mathrm{NH}_{4}\right)_{2} \mathrm{~S}_{2} \mathrm{O}_{8}$, contains peroxy group, is a strong oxidant. Mainly used as oxidant, bleach, disinfectant, photographic materials, analytical reagents, etc. Research shows that APS is widely used in battery industry as oxidant and bleaching agent. Using aniline, citric acid and APS as raw materials, PANI nanometer powder was prepared by in situ polymerization. 

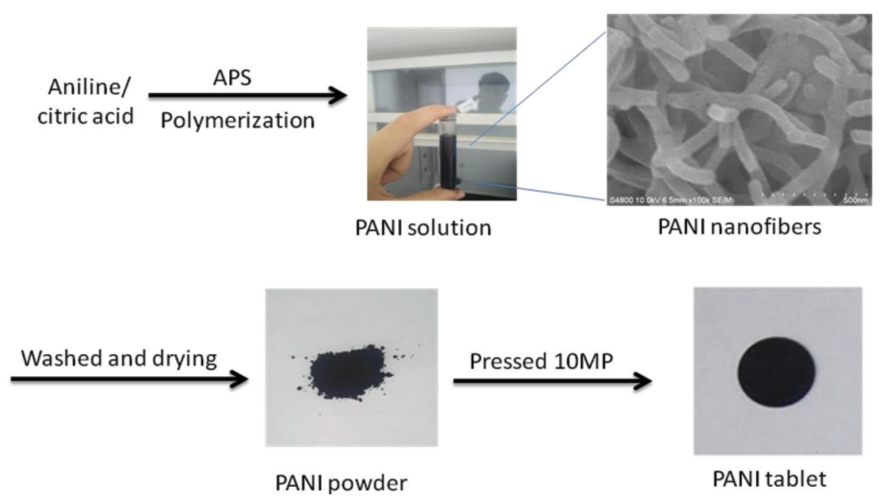

Figure 1 Procedure of the preparation of polyaniline powders: PANI 1-6.

A typical experimental procedure in Figure 1 was as follows: The PANI-citric acid was synthesized by using $5 \mathrm{~g}$ of aniline, $5 \mathrm{~g}$ of APS and $1 \mathrm{~g}$ of citric acid in $400 \mathrm{~mL}$ water at room temperature. The prepared PANI-citric acid was washed three times with ethanol and deionized water. To understand the influence of doping on crystallization of PANI, a set of doping control experiments were performed with mass ratio (aniline:citric acid) of 7:1, 6:1, 5:1, 5:2, 5:3, 5:4, corresponding to sample "PANI 1-6" as shown in Figure 1, respectively, while the other synthetic parameters were kept constant. After drying of black PANI powders, pressed PANI plates are acquired at pressures about $10 \mathrm{MPa}$.

A portion of the 5:3 (aniline:citric acid), with the highest conductivity PANI powder PANI-5 $\left(3.289 \times 10^{-2} \mathrm{~S} / \mathrm{cm}\right)$ was selected as raw material for inks. $1 \mathrm{~g}$ of PANI powder, $0.1 \mathrm{~g}$ of Polystyrene Sulfonate (PSS) and $0.1 \mathrm{~g}$ of Polyethylene Glycol (PEG) were mixed into a paste of black inks (Figure 7). A set of re-doping control experiments were performed with mass ratio (PANI:PSS) of 10:1, 10:2, 10:3, 10:4, 10:5, 10:6 in Figure 7, corresponding to sample "Ink 1-6", respectively, while the other synthetic parameters were kept constant.

SEM studies by using a FEI-Sirion200 Scanning SEM, PANI powder solutions were drop-cast on the silicon substrates followed by drying for $12 \mathrm{~h}$ at $60^{\circ} \mathrm{C}$. UV-Vis absorption spectra of the samples dispersed in distilled water, through ultrasonic irradiation, were obtained with U-4100 UV spectrophotometer in the range of 200-850 nm. XRD patterns were performed on a XD-6000 X-ray diffractometer using a $\mathrm{Cu}-\mathrm{K} \alpha$ radiation source $(\lambda=1.5418 \AA)$. The conductivity measurements of PANI powder tablet pressed under the pressure of $10 \mathrm{MPa}$ were performed with the four-line probe approach by using a CINDBEST CS-6 four probe platform (Sendongbao Co., China) and a CHI660E electrochemical workstation (Shanghai Huachen Co., China), at room temperature. CINDBEST CS-6 four probe platform was used to check conductivity of the ink with microscopic inspection (Figure 1).

\section{RESULTS AND DISCUSSION}

\subsection{Polyaniline Powders: PANI 1-6}

\subsubsection{Characterization of polyaniline}

Figure $2 \mathrm{a}$ and $2 \mathrm{~b}$ shows similar irregular nanoblocks of PANI 1 and 2. Figure $2 \mathrm{c}$ and $2 \mathrm{~d}$ and present mess nanoblocks of PANI-3 and 4 , respectively. With the increase ratio of aniline:citric acid of $5: 3$, uniformed nanofibers with diameter of $50 \mathrm{~nm}$ come out are observed (Figure 2e). Moreover, there nanofiber generate conductive networks. 2D layered nanosheets with the length of $2-3 \mathrm{~nm}$ can orderly self-assemble (Figure 2f). The thickness of the nanosheets is $<50 \mathrm{~nm}$.

Figure 3 presents the UV/Vis spectra of the doped PANI in water. The PANI-citric acid forms a black suspension (powders in Figure 1). Remarkably, these PANI-citric acid nanofibers have similar absorption peaks at 271 and $375 \mathrm{~nm}$ (Figure 3), citric acid is most likely tightly incorporated as anions within the PANI backbone during
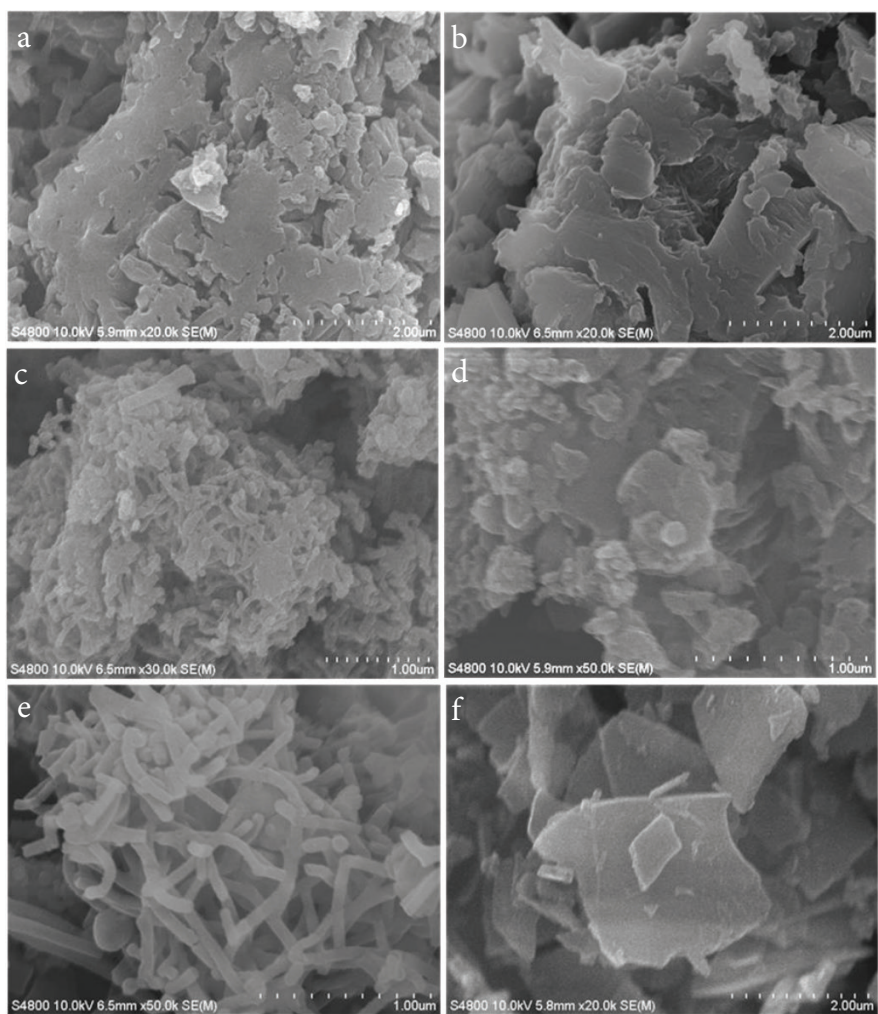

Figure 2 SEM images of PANI with mass ratio of aniline:citric acid: (a) 7:1, (b) 6:1, (c) 5:1, (d) 5:2, (e) 5:3 and (f) 5:4, corresponding to sample PANI 1-6, respectively.

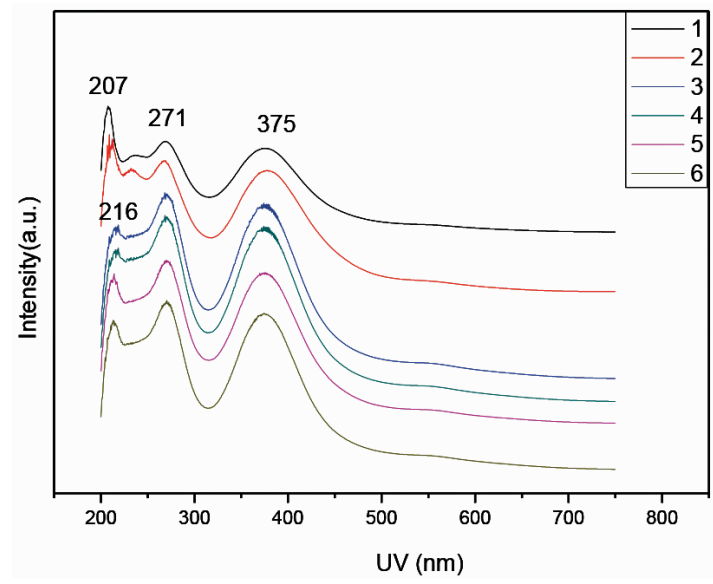

Figure 3 UV/Vis spectra of PANI 1-6 samples doped with citric acid, respectively. 
the in situ polymerization of aniline in citric acid solution. PANI 1 and 2 has absorbs peak $207 \mathrm{~nm}$ while PANI 3-6 absorbs $216 \mathrm{~nm}$.

Figure 4 shows a series of XRD patterns for confirming doping effects on PANI crystallization. It is worthy to mention that high crystalline structures have a similar strong peak at $2 \theta=6.5^{\circ}, 18.5^{\circ}$ and $25.8^{\circ}$. It can be seen that PANI 1-6 have similar high crystallinity (about 70\%). In proper doping level, PANI could crystallized and then self-assembled to form uniform shape and morphology such as $1 \mathrm{D}$ nanofibers by $\pi-\pi$ stacking [17].

The measurement of conductivity is influenced by temperature, electrode constant and alternating current (AC) supply frequency. The measure of an object's ability to conduct electricity is called conductivity $\mathrm{G}$, which is the inverse of the resistance R. The electrochemical workstation tests the conductivity by connecting the four probes of the probe station with the four electrodes of the electrochemical workstation, and then selecting the cyclic voltammetry (CV) test method for testing. Conductivity of PANI-citric acid plates was checked by using a CINDBEST CS- 6 four probe platform and a CHI660E electrochemical workstation, at room temperature. I-V curves identify typical semiconductor conductivity. Table 1 and Figure 5 show all PANI samples show an increased conductivity from 2.06 to $3.289 \times 10^{-2} \mathrm{~S} / \mathrm{cm}$ along with mass ratio (aniline:citric acid) change form 7:1, 6:1, 5:1, 5:2, at last to 5:3. Moreover, the highest conductivity PANI-5 is one kind of nanofibers $3.289 \times 10^{-2} \mathrm{~S} / \mathrm{cm}$ (red dot in Figure 6) rather than nanosheets PANI-6 with the ratio of 5:4. The reason is that nanofibers have $3 \mathrm{D}$ conductive networks while the nanosheets are not organized. Therefore, the PANI nanofibers with the highest conductivity are selected for raw materials of conductive ink.

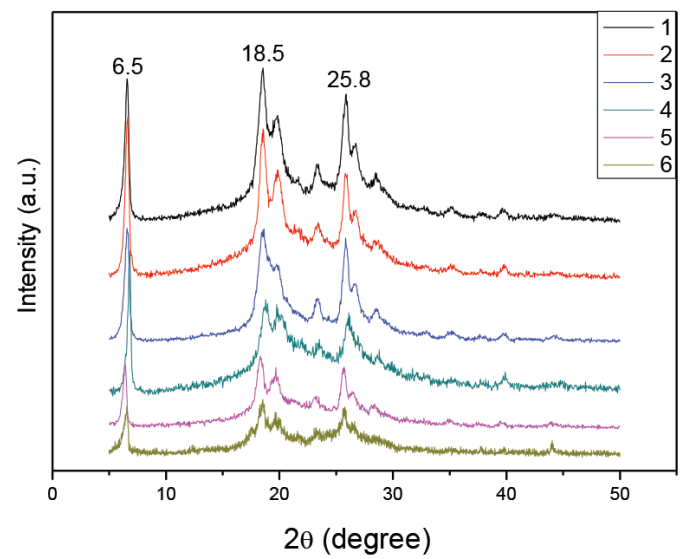

Figure 4 XRD patterns of PANI 1-6 samples doped with citric acid, respectively.

Table 1 Conductivity test data of PANI-citric acid plates

\begin{tabular}{lccccc}
\hline $\begin{array}{l}\text { PANI } \\
\text { samples }\end{array}$ & Aniline (g) & $\begin{array}{c}\text { Mass ratio } \\
\text { (aniline: } \\
\text { citric acid) }\end{array}$ & APS $(\mathbf{g})$ & $\begin{array}{c}\text { Resistivity } \\
\left(\mathbf{1 0}^{\mathbf{2}} \mathbf{\Omega . c m}\right)\end{array}$ & $\begin{array}{c}\text { Conductivity } \\
\left(\mathbf{1 0}^{-\mathbf{2}} \mathbf{S} / \mathbf{c m}\right)\end{array}$ \\
\hline PANI-1 & 5 & $7: 1$ & 5 & 0.485 & 2.061 \\
PANI-2 & 5 & $6: 1$ & 5 & 0.423 & 2.364 \\
PANI-3 & 5 & $5: 1$ & 5 & 0.386 & 2.591 \\
PANI-4 & 5 & $5: 2$ & 5 & 0.364 & 2.747 \\
PANI-5 & 5 & $5: 3$ & 5 & 0.304 & 3.289 \\
PANI-6 & 5 & $5: 4$ & 5 & 0.362 & 2.762 \\
\hline
\end{tabular}

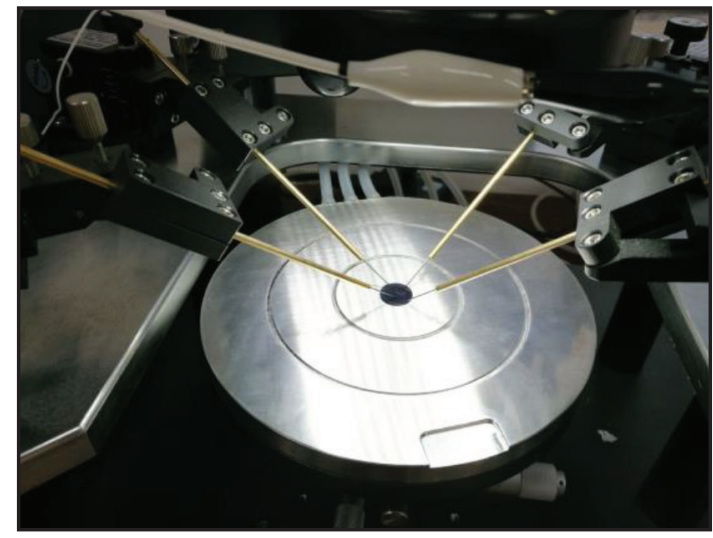

Figure 5 Conductivity test of PANI plates by the four probes platform.

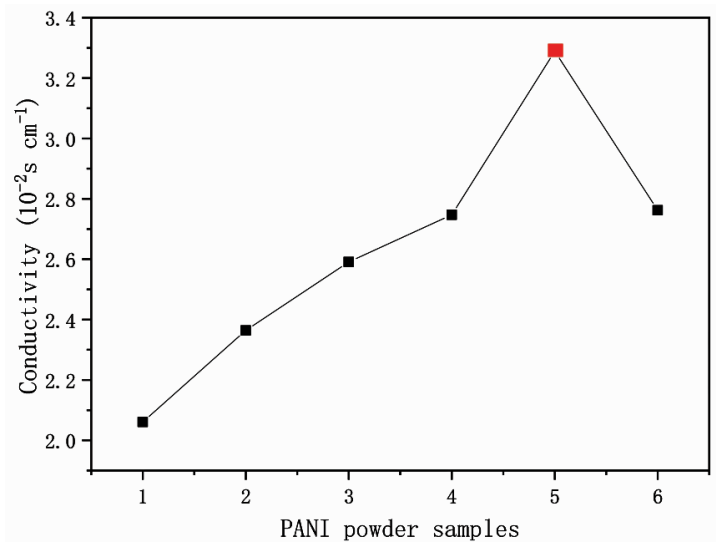

Figure 6 Conductivity of PANI 1-6. The red spot present in PANI-5, which has the highest conductivity of $3.289 \times 10^{-2} \mathrm{~S} / \mathrm{cm}$.

\subsection{Polyaniline Inks: Ink 1-6}

\subsubsection{Characteristics of polyaniline ink}

Figure 7 presents procedure of the preparation of PANI inks. Chinese word “—” (one) was written by using the PANI inks. The smooth surface of inks suggests uniform morphology and size of PANI nanofibers particles. Figure 8 presents PANI inks of Anhui University of Science and Technology university stamp. The inks generate an electronic circuit line where LED light can be turn on.

SEM images show PANI inks in the surface of paper as seen in Figure 9. All PANI inks have the similar morphology with similar size of nanofibers because all PANI particles originate from PANI crystal nanofibers in PANI-5 sample.

The high crystallization rate of PANI nanofibers is beneficial to electron transport and the nanofiber structure of PANI has a higher specific surface area can be evenly dispersed in the ink matrix, which are conducive to the conductive properties of PANI. For deep understanding of the re-doping (PSS acid)/conductivity relationship, PSS content and conductivity of PANI crystal nanofibers doped with different content of PSS acid present in Figure 10, respectively. It can be seen that Ink- 4 re-doped with relative high level of PSS acid $(0.4 \mathrm{~g})$ have higher conductivity $\left(6.49 \times 10^{-3} \mathrm{~S} / \mathrm{cm}\right)$ than that in low level of $<0.3 \mathrm{~g}$ PSS acid. If increase more PSS acid ( $0.6 \mathrm{~g})$ 


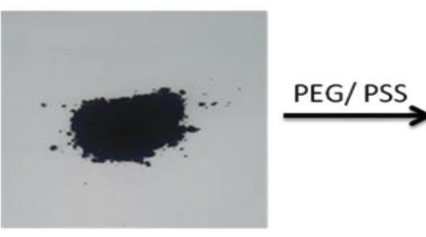

PANI powder

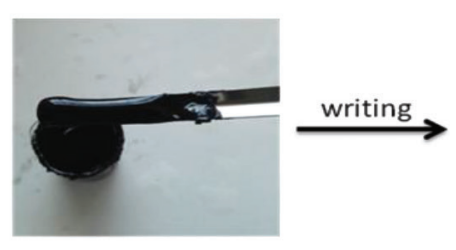

PANI inks

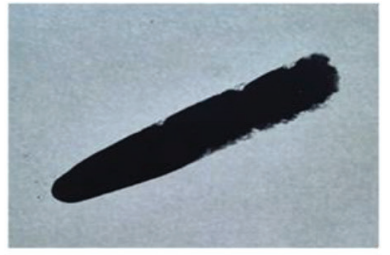

Chinese word "- -"

Figure 7 Procedure of the preparation of PANI inks. Chinese word “一” (one) was written by using the PANI inks.
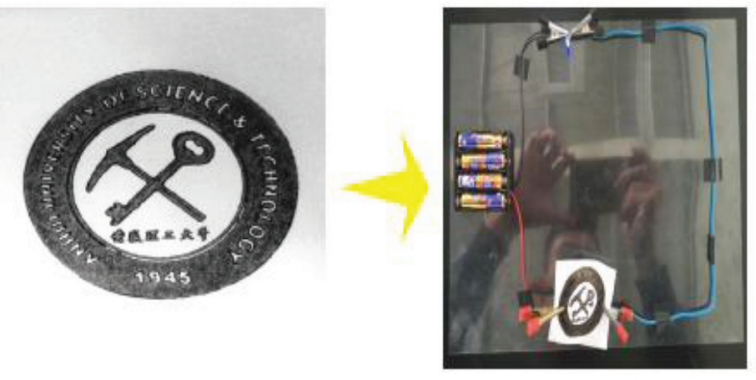

Figure 8 Photograph of circles with PANI inks stamp, which act as electronic circuit line.

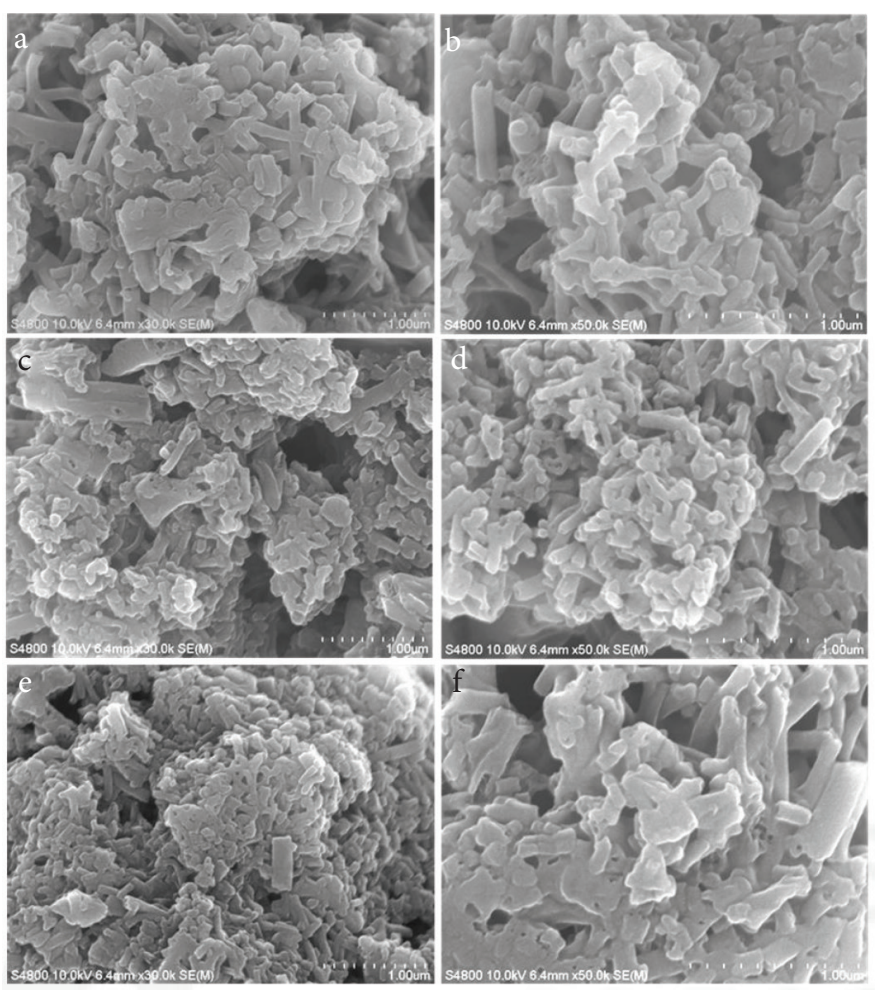

Figure 9 SEM images of (a-f) PANI inks in the surface of paper, corresponding to sample "Ink 1-6", respectively.

in Ink-6, the conductivity decreases to $2.88 \times 10^{-3} \mathrm{~S} / \mathrm{cm}$ (Table 2 ). In fact, only proper doping level of PSS acid induces PANI to re-crystallization and self-assembly. The highest doping level of PSS acid $(0.6 \mathrm{~g})$ is harmful of conductivity. We can draw a conclusion that there is a strong correlation between conductivity and re-doping effect of PSS acid.

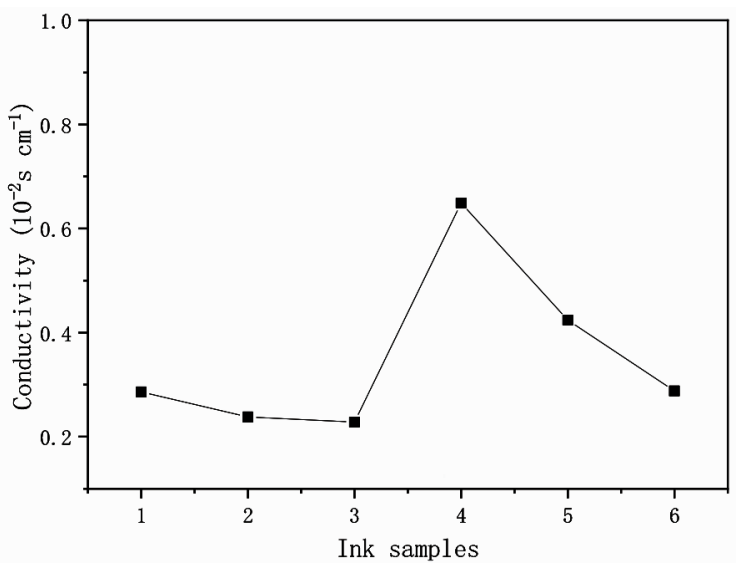

Figure 10 Conductivity of PANI Ink 1-6 samples.

Table 2 The conductivity data of PANI-citric acid inks

\begin{tabular}{lcccccc}
\hline $\begin{array}{l}\text { Ink } \\
\text { samples }\end{array}$ & $\begin{array}{c}\text { Aniline } \\
(\mathbf{g})\end{array}$ & $\begin{array}{c}\text { PSS } \\
(\mathbf{g})\end{array}$ & $\begin{array}{c}\text { PEG } \\
(\mathbf{g})\end{array}$ & $\begin{array}{c}\text { Deionized } \\
\text { water }(\mathbf{g})\end{array}$ & $\begin{array}{c}\text { Resistivity } \\
\left(\mathbf{1 0}^{\mathbf{2}} \mathbf{\Omega . c m}\right)\end{array}$ & $\begin{array}{c}\text { Conductivity } \\
\left(\mathbf{1 0}^{-\mathbf{3}} \mathbf{S} / \mathbf{c m}\right)\end{array}$ \\
\hline Ink-1 & 1 & 0.1 & 0.2 & 2 & 3.50 & 2.86 \\
Ink-2 & 1 & 0.2 & 0.2 & 2 & 4.21 & 2.38 \\
Ink-3 & 1 & 0.3 & 0.2 & 2 & 4.38 & 2.28 \\
Ink-4 & 1 & 0.4 & 0.2 & 2 & 1.54 & 6.49 \\
Ink-5 & 1 & 0.5 & 0.2 & 2 & 2.36 & 4.24 \\
Ink-6 & 1 & 0.6 & 0.2 & 2 & 3.47 & 2.88 \\
\hline
\end{tabular}

\section{CONCLUSION}

In conclusion, we demonstrate a new way to synthesize PANI nanofibers with high crystallinity (The crystallinity can be understood from the doped acid, because of the special structure of citric acid, which promotes crystallization.) and 3D conductive networks. PANI inks mixed with PEG and PSS were prepared and tested by using four probes under the microscope. PANI conductive ink is a kind of new conductive polymer inks, which could be used in electric devices with good electrical conductivity.

\section{CONFLICTS OF INTEREST}

The authors declare they have no conflicts of interest.

\section{AUTHORS' CONTRIBUTION}

$\mathrm{DL}$ is responsible for the writing of the paper and the proposal of the experimental scheme. YL is responsible for the submission and 
the related matters. The other authors are responsible for the experimental optimization and data processing.

\section{ACKNOWLEDGMENTS}

The work is supported by National Natural Science Foundation of China (51775001), Anhui Primary Research and Development Program (1704e1002215), Natural Science Foundation of Anhui Province (1608085MB25), Postdoctoral Science Fund of Anhui Province (2016B108), Anhui Province Key Laboratory of Environmentfriendly Polymer Materials, Anhui University.

\section{REFERENCES}

[1] Yan Y, Wang R, Qiu XH, Wei ZX. Hexagonal superlattice of chiral conducting polymers self-assembled by mimicking $\beta$-sheet proteins with anisotropic electrical transport. J Am Chem Soc 2010;132:12006-12.

[2] Zhong R, Tang Q, Wang S, Zhang H, Zhang F, Xiao M, et al. Self-assembly of enzyme-like nanofibrous G-molecular hydrogel for printed flexible electrochemical sensors. Adv Mater 2018; 30:e1706887.

[3] Qiao Y, Wei Z, Risko C, Li H, Brédas JL, Xu W, et al. Synthesis, experimental and theoretical characterization, and field-effect transistor properties of a new class of dibenzothiophene derivatives: from linear to cyclic architectures. J Mater Chem 2012;22:1313-25.

[4] Soegiarto AC, Comotti A, Ward MD. Controlled orientation of polyconjugated guest molecules in tunable host cavities. J Am Chem Soc 2010;132:14603-16.

[5] Song E, Tortorich RP, da Costa TH, Choi JW. Inkjet printing of conductive polymer nanowire network on flexible substrates and its application in chemical sensing. Microelectron Eng 2015; 145:143-8.

[6] Liao Y, Zhang C, Zhang Y, Strong V, Tang J, Li XG, et al. Carbon nanotube/polyaniline composite nanofibers: facile synthesis and chemosensors. Nano Lett 2011;11:954-9.
[7] Du M, Yao Z, Zhou J, Liu P, Yao T, Yao R. Design of efficient microwave absorbers based on multi-layered polyaniline nanofibers and polyaniline nanofibers $/ \mathrm{Li}_{0.35} \mathrm{Zn}_{0.3} \mathrm{Fe}_{2.35} \mathrm{O}_{4}$ nanocomposite. Synth Met 2017;223:49-57.

[8] Yang TI, Chang SH. Controlled synthesis of metallic iron nanoparticles and their magnetic hyperthermia performance in polyaniline composite nanofibers. Nanotechnology 2017;28:055601.

[9] Wang MS, Wang ZQ, Chen Z, Yang ZL, Tang ZL, Luo HY, et al. One dimensional and coaxial polyaniline@tin dioxide@multiwall carbon nanotube as advanced conductive additive free anode for lithium ion battery. Chem Eng J 2018;334:162-71.

[10] Yi L, Liu L, Guo G, Chen X, Zhang Y, Yu S, et al. Expanded graphite@SnO $@$ @polyaniline composite with enhanced performance as anode materials for lithium ion batteries. Electrochim Acta 2017;240:63-71.

[11] Zuo F, Angelopoulos M, MacDiarmid AG, Epstein AJ. Transport studies of protonated emeraldine polymer: a granular polymeric metal system. Phys Rev B Condens Matter 1987;36:3475-8.

[12] Wessling B, Hiesgen R, Meissner D. STM investigation on primary particle morphology of polyaniline. Acta Polym 1993;44:132-4.

[13] Chang SS, Wu CG. Effects of polymerization media on the nanoscale conductivity and current-voltage characteristics of chemically synthesized polyaniline films. J Phys Chem B 2005; 109:18275-82.

[14] Huang J, Kaner RB. A general chemical route to polyaniline nanofibers. J Am Chem Soc 2004;126:851-5.

[15] Tao Y, Shen Y, Yang L, Han B, Huang F, Li S, et al. Hierarchical self-assembly of hexagonal single-crystal nanosheets into 3D layered superlattices with high conductivity. Nanoscale 2012; 4:3729-33.

[16] Wang Y, Liu J, Tran HD, Mecklenburg M, Guan XN, Stieg AZ, et al. Morphological and dimensional control via hierarchical assembly of doped oligoaniline single crystals. J Am Chem Soc 2012;134:9251-62.

[17] Tao Y, Li J, Xie A, Li S, Chen P, Ni L, et al. Supramolecular self-assembly of three-dimensional polyaniline and polypyrrole crystals. Chem Commun (Cambridge, England) 2014;50: 12757-60. 